Article

\title{
Power-Optimized Sinusoidal Piston Motion and Its Performance Gain for an Alpha-Type Stirling Engine with Limited Regeneration
}

\author{
Mathias Scheunert ${ }^{\circledR}$, Robin Masser, Abdellah Khodja, Raphael Paul, Karsten Schwalbe, \\ Andreas Fischer and Karl Heinz Hoffmann * \\ Institut für Physik, Technische Universität Chemnitz, 09107 Chemnitz, Germany; \\ mathias.scheunert@mailserver.tu-freiberg.de (M.S.); robin.masser@physik.tu-chemnitz.de (R.M.); \\ abdellah.khodja@physik.tu-chemnitz.de (A.K.); raphael.paul@physik.tu-chemnitz.de (R.P.); \\ karsten.schwalbe@physik.tu-chemnitz.de (K.S.); andreas.fischer@physik.tu-chemnitz.de (A.F.) \\ * Correspondence: hoffmann@physik.tu-chemnitz.de
}

Received: 30 July 2020; Accepted: 31 August 2020; Published: 3 September 2020

\begin{abstract}
The recuperation of otherwise lost waste heat provides a formidable way to decrease the primary energy consumption of many technical systems. A possible route to achieve that goal is through the use of Stirling engines, which have shown to be reliable and efficient devices. One can increase their performance by optimizing the piston motion. Here, it is investigated to which extent the cycle averaged power output can be increased by using a special class of adjustable sinusoidal motions (the AS class). In particular the influence of the regeneration effectiveness on the piston motion is examined. It turns out that with the optimized piston motion one can achieve performance gains for the power output of up to $50 \%$ depending on the loss mechanisms involved. A remarkable result is that the power output does not depend strongly on the limitations of the regenerator, in fact-depending on the loss terms-the influence of the regenerator practically vanishes.
\end{abstract}

Keywords: piston motion optimization; endoreversible thermodynamics; stirling engine; irreversibility; power; efficiency; optimization

\section{Introduction}

The global trend towards fossil fuel savings, reduction of $\mathrm{CO}_{2}$ emissions, and use of renewable energy sources is calling for ways to reuse energy and especially to recoup energy in the form of heat, which would be lost otherwise. The Stirling engine, invented by Robert Stirling in 1816 [1,2], is among other devices one of the most suitable engines to harness such waste heat. Owing to its regenerator that internally stores and releases heat during its cyclic operation, it has an excellent theoretical efficiency. It is thus natural that efforts have been undertaken to improve its operation even further, for example via design and process parameter optimizations [3-6] or free-piston approaches $[7,8]$.

A particular interesting way to optimize heat engines in general is the optimization of the piston motion [9-13]. In the past, the piston trajectories of engines with Otto [14-16], Diesel [10,17,18], Miller [19] and Brayton [20] cycles as well as light-driven engines [21-23] have been the subject of interest. These investigations showed substantial improvements of the engine efficiencies and power outputs. Such an approach has also been taken for Stirling engines. Kojima [24] considered the optimal control problem of free-piston Stirling engine generators. Moreover, Craun and Bamieh [25] and Craun [26] solved cyclic optimal control problems for an actuated beta-type Stirling engine, based on the Schmidt model with an indirect optimization method, and based on a higher fidelity reduced order model with a direct optimization method, respectively. 
The aim of the present study is to follow the same route and to determine the potential gains in the power output for a particular type of Stirling engine, namely a Stirling engine in alpha configuration characterized by its two independently operating pistons. While this work was initialized by the task to use Stirling engines to recover waste heat of machine tools-a task which has a considerable energy-saving potential—we here analyze an endoreversible alpha Stirling engine and its optimal piston motion in general. In order to avoid a full control theory based calculation, which would be overly demanding for this preliminary assessment, here a simpler approach is taken [27]. We investigate the gains for a parametrized piston motion, which will provide lower limits for the gain achievable by a control theory based optimization.

While in [27] a Stirling engine with ideal regeneration was analyzed, here our special focus will be on the imperfect regeneration and its consequences.

The goal of our investigations is to go beyond a classical ideal thermodynamic treatment and take important dissipative processes into account. This approach follows the ideas of finite-time thermodynamics [28-31]. One well developed finite-time thermodynamics method, which will be used here, is Endoreversible Thermodynamics. It has proven to be a suitable tool to model dissipative systems, for a review the reader is referred to [32-34]. In particular it provides a methodology to capture the dissipative loss terms quantitatively. For the Stirling engine considered here these include losses due to the mechanical friction of the moving pistons, the flow friction of the working gas flowing through the regenerator while moving from the hot to the cold cylinder and back, and the finite heat transfer rates from the heat source into the engine and from the engine into the heat sink.

In Endoreversible Thermodynamics, the Stirling engine is seen as a collection of subsystems. While these are still reversible solely, the connections between them can be irreversible. The advantage of this description is that knowledge about equilibrium systems can be applied and entropy production only occurs through transfers between the subsystems. Initially the focus of Endoreversible Thermodynamics was on relatively simple heat engines and refrigerators [35-38], but its scope widened later to include applications related to for instance solar power [39-42] and chemical processes [15,43-46]. Also the performance of more complex systems [47-52] has been investigated and recent work widened the treatment to the thermodynamics of computing [53] and systems under temporal fluctuations [54] leading to generalizations of the well known Curzon-Ahlborn efficiency [55].

\section{The AS Class of Piston Motions}

Typically in classical Stirling engine modeling, time-harmonic piston motions with a fixed phase shift $\Delta \phi=-\pi / 2$ between the two cylinders are considered.

Then for a single cylinder its change in volume, which will be called "standard harmonic motion" (ST), is described by

$$
V(t)=V_{\text {dead }}+\Delta V\left(1+\sin \left(2 \pi t / t_{0}\right)\right) / 2
$$

Therein, $V_{\text {dead }}$ denotes the dead volume, i.e., the minimum volume that is left unswept by the piston, $\Delta V$ describes the displacement, i.e., the volume that is swept by the piston during its motion, and $t_{0}$ is the period of motion. As the goal is to maximize the average power output compared to the classical Stirling engine motion by the use of a nonlinear optimization approach, we introduce a new motion class for the time-dependent volume characterized by three main properties. First, the function allows to vary both the volume "speed" $\dot{V}(t)$ and the time the volume spends close to its maximum $V_{\text {dead }}+\Delta V$ or close to its minimum $V_{\text {dead }}$. Second, it keeps the periodicity of the ST and, in addition, is able to regain the ST by the proper choice of the function parameters. Third, the function is 
continuously differentiable with respect to time. We define this so-called "adjustable sinusoidal motion" (AS)

$$
V(t)=V_{\text {dead }}+\Delta V f\left(t / t_{0} ; \sigma, \delta\right),
$$

the core of which consists of the function composition

$$
f(x ; \sigma, \delta)=\left(f_{1} \circ f_{2}\right)(x)=f_{1}\left(f_{2}(x ; \delta) ; \sigma\right),
$$

with $f_{1}$ as

$$
f_{1}(y ; \sigma)=(\sin (2 \pi y+\sigma \sin (4 \pi y))+1) / 2,
$$

and $f_{2}$ as

$$
f_{2}(x ; \delta)=x+\delta(1-\cos (2 \pi x)) .
$$

Like in the ST, $V_{\text {dead }}$ and $\Delta V$ are the dead volume and the displacement, respectively. The period of motion is chosen to be $t_{0}=0.1 \mathrm{~s}$ for the remainder of the study.

Let us now focus on the dimensionless scalar motion control parameter $\sigma$ and $\delta$. One can clearly see that by choosing $\sigma=\delta=0$, the AS turns into the ST, i.e., the standard harmonic motion can be considered a special case of the adjustable sinusoidal motion class. Keeping $\delta=0$, the alteration of the shape of the AS with respect to the parameter $\sigma$ is shown in Figure 1.

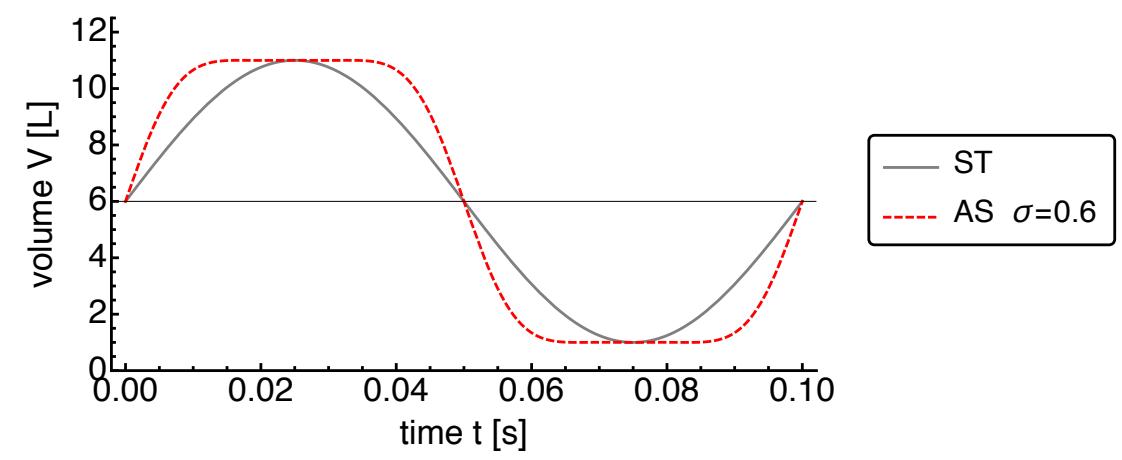

Figure 1. Comparison of the cylinder volume over time $V(t)$ for the "standard harmonic motion" ST (solid line) and "adjustable sinusoidal motion" AS (dashed line) for $t_{0}=0.1 \mathrm{~s}$ with $\delta=0$ and $\sigma=0.6$. For the AS motion with $\sigma>0$, the maximum volume speed (e.g., around $t=0.05 \mathrm{~s}$ ) increases, while the piston speed around the volume extrema decreases.

An increase of $\sigma$ extends the dwell time of the piston around the minimum and maximum volume and it also increases the piston speed within the phases of compression and expansion.

A further shape change of the AS motion due to an additional variation of parameter $\delta$ is displayed in Figure 2.

This parameter affects the symmetry of the shape of the volume vs. time curve within a period of motion. For $\delta>0$, the dwell time of the piston at the volume maximum is reduced, while the dwell time around the volume minimum is increased. As Figure 2 shows, the slope of the volume curve is practically unchanged while varying $\delta$, thus it has a much smaller influence on the piston speed than the parameter $\sigma$. 


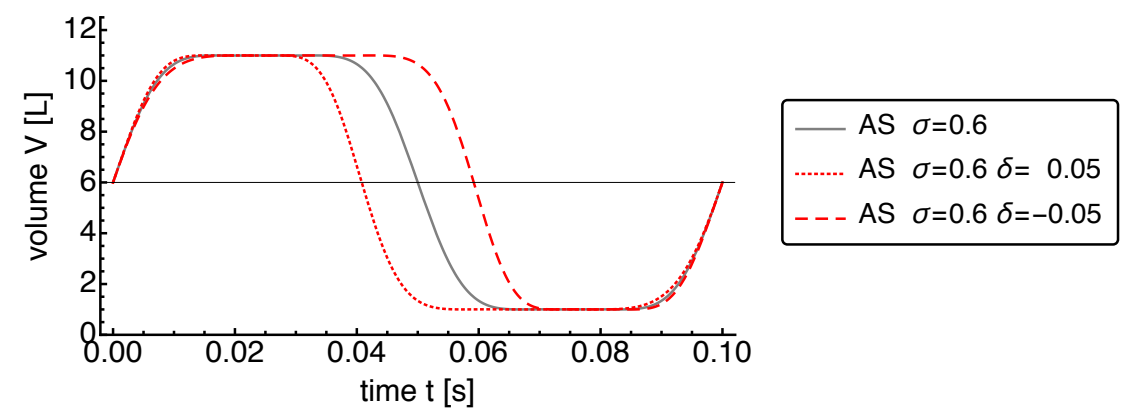

Figure 2. Plot of the cylinder volume over time $V(t)$ for the "adjustable sinusoidal motion" AS for $t_{0}=0.1 \mathrm{~s}$ with $\delta=0$ and $\sigma=0.6$ (solid line) in comparison to the AS motion with $\delta=-0.05$ and $\sigma=0.6$ (dashed line) and with $\delta=0.05$ and $\sigma=0.6$ (dotted line). Within a period of motion, negative values of $\delta$ reduce the time the volume spends around its maximum and increase the time it spends around its minimum. Positive values lead to the opposite effect.

In view of the optimization of the AS, constraints for the parameter $\sigma:-0.13<\sigma<0.6$ and $\delta:-0.08<\delta<0.08$ are introduced, which have proven useful in the course of the performed numerical studies. Additionally, we no longer consider the piston motion of a single cylinder but rather take into account the entire dynamics of the alpha-type Stirling engine. This is characterized by the two piston motions

$$
\begin{aligned}
& V_{1}(t)=V_{\text {dead }}+\Delta V f\left(t / t_{0} ; \sigma_{1}, \delta_{1}\right), \\
& V_{2}(t)=V_{\text {dead }}+\Delta V f\left(t / t_{0}+\Delta t / t_{0} ; \sigma_{2}, \delta_{2}\right),
\end{aligned}
$$

that are coupled by the time shift $\Delta t / t_{0}$. The ST value for this parameter translates into $\Delta t=-0.25 t_{0}$ corresponding to $\Delta \phi=-\pi / 2$. Hence, the subsequent analysis of the potential power gains achievable by the nonlinear optimization of the piston dynamics incorporates five dimensionless parameters $\sigma_{1}$, $\delta_{1}, \sigma_{2}, \delta_{2}$, and $\Delta t$. Once these have been optimized for a particular engine, the optimized function for the volume will be labeled by "optimized sinusoidal motion" OS.

\section{Endoreversible Thermodynamics}

Endoreversible modeling aims at a description of a system by specifying the system components and the processes occurring in form of equilibrium subsystems and reversible or irreversible interactions, respectively. Endoreversible Thermodynamics has shown to be a reliable modeling tool within the context of finite-time thermodynamics and has been applied in a variety of systems.

The subsystems needed in our application come in two varieties: either as (in)finite Reservoirs or Engines. The latter serve for energy conversion, while the former store extensities and energy. A finite Reservoir is defined by specifying its energy $E_{i}$ as a function of its extensities, where $i$ denotes the $i$-th subsystem. For each extensity $X_{i}^{\alpha}$ a corresponding intensity $Y_{i}^{\alpha}$ can be calculated by

$$
Y_{i}^{\alpha}=\frac{\partial E_{i}\left(X_{i}^{\alpha}\right)}{\partial X_{i}^{\alpha}}
$$

where the superscript $\alpha$ specifies the extensity. For example the chemical potential $\mu=Y^{n}$, as it corresponds to the extensity mole number $n$. Then a change in energy can be expressed by changes in the extensities

$$
\mathrm{d} E_{i}=\sum_{\alpha} Y_{i}^{\alpha} \mathrm{d} X_{i}^{\alpha}
$$


As a consequence each flux of extensity $J_{i}^{\alpha}$ carries an accompanying flux of energy

$$
I_{i}^{\alpha}=Y_{i}^{\alpha} J_{i}^{\alpha}
$$

into the subsystem.

For each subsystem $i$ all extensities including the energy can then be accounted for by balance equations

$$
\begin{aligned}
\frac{\mathrm{d}}{\mathrm{d} t} X_{i}^{\alpha} & =\sum_{k} J_{i, k}^{\alpha} \quad \text { for all } \alpha \text { and } \\
\frac{\mathrm{d}}{\mathrm{d} t} E_{i} & =\sum_{k, \alpha} I_{i, k}^{\alpha}
\end{aligned}
$$

where $k$ is the contact point of subsystem $i$, which is here labeled by the interaction they are connected to. For an infinite reservoir like a fixed temperature heat bath its intensity does not change if the corresponding extensity is added or removed, and thus it is specified by its fixed intensity.

The endoreversible subsystem class Engines differs from Reservoirs in that they can neither store extensities nor energy. Thus all extensity fluxes and energy fluxes going in and out of an interaction have to cancel:

$$
\begin{aligned}
& 0=\sum_{k} J_{i, k}^{\alpha} \text { for all } \alpha \text { and } \\
& 0=\sum_{k, \alpha} I_{i, k}^{\alpha} .
\end{aligned}
$$

The interactions between subsystems are the important entities for the non-equilibrium description of the overall system. They are the entities in which entropy can be produced and are thus indispensable for modeling irreversibilities. Interactions are described by specifying the extensity and energy fluxes at all connected contact points as functions of the state variables of the subsystems. Due to Equation (10) it suffices to specify at each contact point $k$ of subsystem $i$ either the extensity flux $J_{i, k}^{\alpha}$ or the energy flux $I_{i, k}^{\alpha}$. Usually such interactions contain transport parameters like a heat conductance, which specifies the heat flux between to contact points in terms of the temperature difference. Nonetheless, interactions can also be reversible. In that case the flux of extensity $\alpha$ is specified by the requirement that equal intensities of the connected subsystems $Y_{i}^{\alpha}=Y_{j}^{\alpha}$ have to be maintained. Within one interaction for all conserved extensities the respective fluxes have to balance at all times, but entropy can be produced. For simplicity it is sometimes possible to not consider the carrying extensity of an energy flux and just use the latter referred to as power flux to describe the rate of transferred energy.

\section{The Stirling Engine Model}

\subsection{The Structure of the Endoreversible Stirling Engine Model}

The endoreversible description just introduced enables us to build the model of the alpha-type Stirling engine as shown in Figure 3. Here, the subsystems shown as circles are the endoreversible engines $\mathrm{T} 1$ and $\mathrm{T} 2$ representing the mechanical transmission. They are the link between the cylinders of the Stirling engine (reservoirs 1 and 2) and the environment (reservoirs E) and convert the volume work flux of the piston stroke into power fluxes. The latter are collected in the work reservoir WT for net power from the volumetric process and in the work reservoir WF for frictional power loss of the mechanical transmission.

The reservoirs $\mathrm{H}$ and $\mathrm{C}$ represent the hot and the cold heat bath, respectively. They are irreversibly connected to reservoirs 1 and 2 which are used to model the gas within the engine's hot and cold cylinder. Finally, the irreversible interactions between them, which are further connected to the 
work and entropy reservoirs WR and SR, respectively, are used to model the regenerator of the Stirling engine.

The following subsections provide a more detailed description of the reservoirs and interactions.

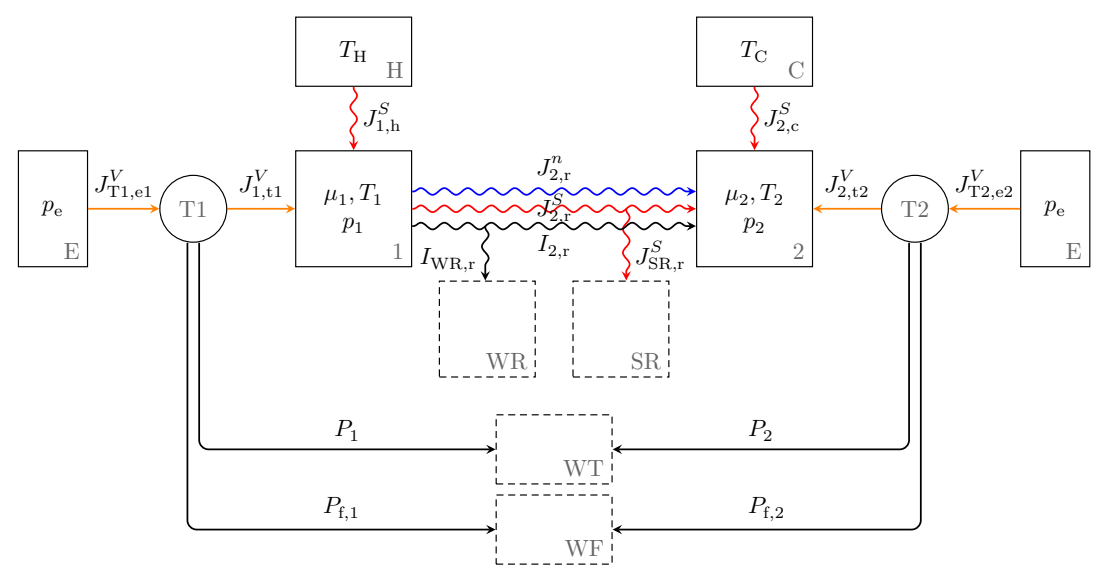

Figure 3. Endoreversible model of the alpha-type Stirling engine with reservoirs (rectangles), engines (circles), and reversible (straight lines) as well as irreversible (wavy lines) interactions. The dashed rectangles represent reservoirs, which are not physically existent but which primarily serve bookkeeping purposes. At the top, there are the hot and the cold heat baths $\mathrm{H}$ and C, respectively, which are irreversibly connected to the hot and cold cylinder 1 and 2 of the Stirling engine. The regenerator between these cylinders is modeled as an irreversible interaction. Also, for bookkeeping purposes, this interaction is connected to a work reservoir WR and an entropy reservoir SR. The endoreversible engines $\mathrm{T} 1$ and $\mathrm{T} 2$ represent the mechanical transmission the pistons are connected to. They further interact with the environment, which is modeled as a (distributed) volume reservoir E. The work reservoirs WT and WF collect the net power and the friction losses, respectively, from the mechanical transmission engines.

\subsection{The Working Fluid}

The working fluid in the two cylinders of the Stirling engine is modeled as a diatomic ideal gas with the thermal equation of state

$$
p V=n R T,
$$

where $p, V, n, R$ and $T$ are the pressure, volume, mole number, gas constant and temperature, respectively. The caloric equation of state is

$$
U=\hat{c}_{V} n R T
$$

where $U$ is the internal energy and $\hat{c}_{V}$ is the dimensionless specific heat capacity at constant volume, which is here chosen as $\hat{c}_{V}=5 / 2$. As the momentum and the angular momentum of the gas are not considered here, the energy $E$ from Equation (9) is here identical to the internal energy $U$.

For an ideal gas the usual extensities are the entropy $S, V$ and $n$, and once the internal energy is known as a function of those one can easily get all the intensities. Due to the presence of multi-extensity fluxes [56] it turns out to be advantageous to use $U, V$ and $n$ instead, and express the other thermodynamic variables by these. We find

$$
S(U, V, n)=n R\left(\hat{c}_{V} \ln \frac{U}{U_{0}}+\ln \frac{V}{V_{0}}-\left(1+\hat{c}_{V}\right) \ln \frac{n}{n_{0}}\right)+n \frac{S_{0}}{n_{0}}
$$


with $U_{0}, V_{0}$ and $n_{0}$ being the reference internal energy, volume and mole number, respectively, for the reference entropy $S_{0}\left(U_{0}, V_{0}, n_{0}\right)$. For the intensities one obtains

$$
\begin{aligned}
T(U, V, n) & =\frac{U}{\hat{c}_{V} n R}, \\
p(U, V, n) & =\frac{U}{\hat{c}_{V} V}, \\
\mu(U, V, n) & =\frac{U}{\hat{c}_{V} n}\left(1+\hat{c}_{V}+\left(1+\hat{c}_{V}\right) \ln \frac{n}{n_{0}}-\hat{c}_{V} \ln \frac{U}{U_{0}}-\ln \frac{V}{V_{0}}-\frac{S_{0}}{n_{0} R}\right) .
\end{aligned}
$$

\subsection{Heat Transfer}

The heat transfer between the hot heat bath $\mathrm{H}$ and the gas reservoir 1 as well as between the gas reservoir 2 and the cold heat bath $C$ are represented by two interactions $h$ and c, respectively. Both are assumed to be Newtonian, and thus, with the hot and cold heat bath temperatures $T_{\mathrm{H}}$ and $T_{\mathrm{C}}$, one obtains

$$
\begin{gathered}
I_{1, \mathrm{~h}}^{S}=\kappa\left(T_{\mathrm{H}}-T_{1}\right)=-I_{\mathrm{H}, \mathrm{h}}^{S}, \\
I_{2, \mathrm{c}}^{S}=\kappa\left(T_{\mathrm{C}}-T_{1}\right)=-I_{\mathrm{C}, \mathrm{c}}^{S},
\end{gathered}
$$

where $\kappa$ is the heat conductance. The accompanying entropy fluxes into or out of the reservoirs are

$$
\begin{gathered}
J_{1, \mathrm{~h}}^{S}=I_{1, \mathrm{~h}}^{S} / T_{1}, \\
J_{2, \mathrm{c}}^{S}=I_{2, \mathrm{c}}^{S} / T_{2} .
\end{gathered}
$$

The temperatures of both heat baths remain constant throughout the operation of the Stirling engine at $T_{\mathrm{H}}$ and $T_{\mathrm{C}}$, respectively.

\subsection{The Imperfect Regenerator}

A real regenerator stores energy and entropy at a continuum of temperatures. The possible temperature range is determined by the temperatures of the reservoirs 1 and 2 . In cyclic operation gas streams from one cylinder into the other and back. While flowing from the hot cylinder 1 along its path through the regenerator the gas temperature will drop, but due to finite heat transfer it will stay above the local regenerator temperature. Finally it will leave the regenerator at a temperature still above the cold cylinder 2 temperature. On the way back it will start below the regenerator temperature and stay below it. It will warm up but will not reach the hot cylinder temperature. This necessitates the discussion of two cases depending on the flow direction of the gas.

The regenerator performance depends on a variety of features and is degraded by several different loss phenomena. In the theories of Martini as well as Kühl and Schulz for example, these losses are referred to as the reheat loss, the temperature swing loss, the thermal conduction loss, the internal temperature swing loss, the dispersion loss, and the bypass loss [57].

However, what is essential from a thermodynamic point of view is the thermodynamic state of the gas when it enters and leaves the regenerator at its contacts to the cylinders. We will thus concentrate on these properties, and make the simplifying assumption, that the gas amount in the regenerator can be neglected compared to the gas amounts in the cylinders.

The imperfect regenerator is here modeled as an irreversible interaction between reservoirs 1 and 2 and two bookkeeping reservoirs, which provide the storage for energy WR and entropy SR. This regenerator interaction is labeled with " $r$ ". We define the regenerator interaction by giving all the fluxes at the contact points it connects to. The gas flow is a multi-extensity flux [56], which transports particles (mols) and entropy $(\alpha=S n)$. 
The particle fluxes are specified as:

$$
\begin{aligned}
& J_{1, \mathrm{r}}^{n}=\alpha\left(p_{2}-p_{1}\right), \\
& J_{2, \mathrm{r}}^{n}=\alpha\left(p_{1}-p_{2}\right),
\end{aligned}
$$

where $\alpha$ is the mass transfer coefficient. As both particle fluxes have the same magnitude and differ only in their sign, $J_{1, \mathrm{r}}^{n}=-J_{2, \mathrm{r}}^{n}$, no gas is lost in the regenerator. If $J_{i, \mathrm{r}}^{n}$ is positive, gas flows into reservoir $i$, if it is negative gas flows out of reservoir $i$. Thus, the sign of $J_{i, \mathrm{r}}^{n}$ can be used to discriminate between the two flow cases mentioned above. If needed, fluxes of this interaction will be split into two summands

$$
\begin{aligned}
& J_{i, \mathrm{r}}^{\alpha}=J_{\rightarrow i, \mathrm{r}}^{\alpha}+J_{\leftarrow i, \mathrm{r}}^{\alpha}, \\
& I_{i, \mathrm{r}}^{\alpha}=I_{\rightarrow i, \mathrm{r}}^{\alpha}+I_{\leftarrow i, \mathrm{r}}^{\alpha},
\end{aligned}
$$

where $J_{\rightarrow i, \mathrm{r}}^{\alpha}$ and $I_{\rightarrow i, \mathrm{r}}^{\alpha}$ indicate that $J_{i, \mathrm{r}}^{n}$ is positive and flows into reservoir $i$, while $J_{\leftarrow i, \mathrm{r}}^{\alpha}$ and $I_{\leftarrow i, \mathrm{r}}^{\alpha}$ indicate that $J_{i, \mathrm{r}}^{n}$ is negative and flows out of reservoir $i$. With this notation we set

$$
I_{\leftarrow 1, \mathrm{r}}^{S n}= \begin{cases}J_{1, \mathrm{r}}^{n} h_{1} & \text { if } \quad J_{1, \mathrm{r}}^{n}<0, \\ 0 & \text { otherwise, }\end{cases}
$$

with $h_{i}=H_{i} / n_{i}$ being the molar enthalpy of subsystem $i$ and

$$
I_{\rightarrow 1, \mathrm{r}}^{S n}= \begin{cases}J_{1, \mathrm{r}}^{n}\left(r_{1} h_{1}+\left(1-r_{1}\right) h_{2}\right) & \text { if } J_{1, \mathrm{r}}^{n}>0, \\ 0 & \text { otherwise }\end{cases}
$$

where $r_{1}$ is the regeneration coefficient for reservoir 1 . Likewise we set

$$
I_{\leftarrow 2, \mathrm{r}}^{S n}= \begin{cases}J_{2, \mathrm{r}}^{n} h_{2} & \text { if } J_{2, \mathrm{r}}^{n}<0, \\ 0 & \text { otherwise, }\end{cases}
$$

and

$$
I_{\rightarrow 2, \mathrm{r}}^{S n}= \begin{cases}J_{2, \mathrm{r}}^{n}\left(r_{2} h_{2}+\left(1-r_{2}\right) h_{1}\right) & \text { if } J_{2, \mathrm{r}}^{n}>0, \\ 0 & \text { otherwise }\end{cases}
$$

where $r_{2}$ is the regeneration coefficient for reservoir 2 .

This choice corresponds to the physical situation in the regenerator described above: The gas leaving one of the cylinders has the properties of the gas in the cylinder it comes from leading to Equations (29) and (31). For the gas entering one of the cylinders the properties of the gas depend on the extent of the regeneration. If no regeneration would take place $\left(r_{i}=0\right)$, this gas would enter with exactly the properties of the cylinder it came from. If regeneration is present, then the gas properties would become closer and closer to the properties of the gas within the receiving cylinder with increasing $r_{i}$, completely reaching the properties of the receiving cylinder for $r_{i}=1$. This leads to Equations (30) and (32).

Given the energy fluxes $I_{1, \mathrm{r}}^{S n}$ and $I_{2, \mathrm{r}}^{S n}$, the entropy fluxes can be inferred from $I_{i, \mathrm{r}}^{S n}=T_{i} J_{i, \mathrm{r}}^{S}+\mu_{i} J_{i, \mathrm{r}}^{n}$ and one finds

$$
J_{i, \mathrm{r}}^{S}=\left(I_{i, \mathrm{r}}^{S n}-\mu_{i} J_{i, \mathrm{r}}^{n}\right) / T_{i}
$$


In case of out going particle fluxes this leads to

$$
\begin{aligned}
& J_{\leftarrow 1, \mathrm{r}}^{S}=s_{1} J_{1, \mathrm{r}}^{n}, \\
& J_{\leftarrow 2, \mathrm{r}}^{S}=s_{2} J_{2, \mathrm{r}}^{n},
\end{aligned}
$$

where $s_{i}=S_{i} / n_{i}$ is the molar entropy of subsystem $i$, in the other case one has to use Equation (33). Finally, the fluxes into the bookkeeping reservoirs WR and SR are specified:

$$
\begin{aligned}
I_{\mathrm{WR}, \mathrm{r}} & =-I_{1, \mathrm{r}}^{S n}-I_{2, \mathrm{r}}^{S n} \quad \text { and } \\
J_{\mathrm{SR}, \mathrm{r}}^{S} & =-J_{1, \mathrm{r}}^{S}-J_{2, \mathrm{r}}^{S} .
\end{aligned}
$$

If we now look at the energy content $E_{\mathrm{WR}}(t)$ of reservoir WR, then it turns out that

$$
E_{\mathrm{WR}}\left(t_{0}\right)-E_{\mathrm{WR}}(0)=\int_{0}^{t_{0}} I_{\mathrm{WR}, \mathrm{r}} \mathrm{d} t .
$$

A cyclic operation requires reservoir WR to return to its initial state at the end of the cycle, $E_{\mathrm{WR}}(0)=E_{\mathrm{WR}}\left(t_{0}\right)$. This couples the regeneration coefficients $r_{1}$ and $r_{2}$, because in

$$
0=E_{\mathrm{WR}}\left(t_{0}\right)-E_{\mathrm{WR}}(0)=-\int_{0}^{t_{0}}\left(I_{\leftarrow 1, \mathrm{r}}^{S n}+I_{\leftarrow 2, \mathrm{r}}^{S n}+I_{\rightarrow 1, \mathrm{r}}^{S n}+I_{\rightarrow 2, \mathrm{r}}^{S n}\right) \mathrm{d} t
$$

the last two terms in the integral depend on $r_{1}$ and $r_{2}$.

Thus the operation of the above defined regenerator-which we will term an r-Regenerator from here on-will depend on one variable $r$ only: For a given value of the regeneration coefficient $r$, the regeneration coefficients $r_{1}$ and $r_{2}$ are determined in a way such that in stationary cyclic operation (all state variables are cyclic including the energy of WR) the conditions $r=\left(r_{1}+r_{2}\right) / 2$ and Equation (39) are both fulfilled.

Finally, we look at the entropy content in the bookkeeping reservoir SR

$$
S_{\mathrm{SR}}\left(t_{0}\right)-S_{\mathrm{SR}}(0)=\int_{0}^{t_{0}} J_{\mathrm{SR}, \mathrm{r}}^{S} \mathrm{~d} t=-\int_{0}^{t_{0}}\left(J_{1, \mathrm{r}}^{S}+J_{2, \mathrm{r}}^{S}\right) \mathrm{d} t
$$

It allows to determine the cycle averaged regeneration-related entropy production rate

$$
\frac{1}{t_{0}} \int_{0}^{t_{0}}\left(J_{1, \mathrm{r}}^{S}+J_{2, \mathrm{r}}^{S}\right) \mathrm{d} t=-\frac{S_{\mathrm{SR}}\left(t_{0}\right)-S_{\mathrm{SR}}(0)}{t_{0}},
$$

which is part of the overall entropy production rate $\Sigma$ introduced later.

\subsection{The Dynamics}

The temporal development of the system is described in terms of a coupled system of differential equations. From the balance equations for the extensities derived above, the transport laws, and the gas properties we obtain a coupled system of differential equations to be integrated:

$$
\begin{array}{ll}
\dot{U}_{1}=I_{1, \mathrm{~h}}^{S}+I_{1, \mathrm{r}}^{S n}=\kappa\left(T_{\mathrm{H}}-T_{1}\right)+I_{1, \mathrm{r}}^{S n} & \dot{U}_{2}=I_{2, \mathrm{c}}^{S}+I_{2, \mathrm{r}}^{S n}=\kappa\left(T_{\mathrm{C}}-T_{2}\right)+I_{2, \mathrm{r}}^{S n}, \\
V_{1}=V_{1}\left(t ; \sigma_{1}, \delta_{1}\right), & V_{2}=V_{2}\left(t+\Delta t ; \sigma_{2}, \delta_{2}\right), \\
\dot{n}_{1}=J_{1, \mathrm{r}}^{n}=\alpha\left(p_{2}-p_{1}\right), & \dot{n}_{2}=J_{2, \mathrm{r}}^{n}=\alpha\left(p_{1}-p_{2}\right) .
\end{array}
$$




\subsection{Power Output, Efficiency and Entropy Production}

The power output of the Stirling engine originates from the cyclic volume fluxes $J_{\mathrm{T} 1, \mathrm{e} 1}^{V}=J_{1, \mathrm{t} 1}^{V}=\dot{V}_{1}$ and $J_{\mathrm{T} 2, \mathrm{e} 2}^{V}=J_{2, \mathrm{t} 2}^{V}=\dot{V}_{2}$ between cylinders 1 and 2 and the environment over a pressure difference of $p_{1}-p_{\mathrm{e}}$ and $p_{2}-p_{\mathrm{e}}$, respectively. This power flux is delivered by the two engines $\mathrm{T} 1$ and $\mathrm{T} 2$, and then split into fluxes $P_{\mathrm{f}, i}$ and $P_{i}$. The first one is the power lost into friction and here modeled as

$$
P_{\mathrm{f}, i}=\beta \dot{V}_{i}^{2}
$$

where $\beta$ is the mechanical friction coefficient [47]. For both cylinders 1 and 2 it is later dissipated to heat dumped into the environment at $T_{\mathrm{C}}$. The usable parts $P_{1}$ and $P_{2}$ with

$$
P_{i}=p_{i} \dot{V}_{i}-P_{\mathrm{f}, i}
$$

are combined into the cycle averaged power output of the Stirling engine

$$
P_{\text {out }}=\frac{1}{t_{0}} \int_{0}^{t_{0}} P_{1}+P_{2} \mathrm{~d} t
$$

The cycle averaged heat fluxes are

$$
q_{\mathrm{H}}=\frac{1}{t_{0}} \int_{0}^{t_{0}} I_{\mathrm{H}, \mathrm{h}}^{S} \mathrm{~d} t \quad q_{\mathrm{C}}=\frac{1}{t_{0}} \int_{0}^{t_{0}} I_{\mathrm{C}, \mathrm{c}}^{S} \mathrm{~d} t,
$$

and the efficiency is $\eta=P_{\text {out }} / q_{\mathrm{H}}$. Finally the cycle averaged entropy production rate $\Sigma$ can be determined by the entropy changes in the two heat baths $\mathrm{H}$ and $\mathrm{C}$, as-due to their cyclic operation-all other subsystems are after one cycle back into their initial state, i.e., their entropy is unchanged. Thus one finds

$$
\Sigma=\frac{q_{\mathrm{H}}}{T_{\mathrm{H}}}+\frac{q_{\mathrm{C}}}{T_{\mathrm{C}}}+\frac{\int_{0}^{t_{0}} P_{\mathrm{f}, 1}+P_{\mathrm{f}, 2} \mathrm{~d} t}{t_{0} T_{\mathrm{C}}} .
$$

\section{Results}

In this study the aim is to quantify the potential gains in the power output of an alpha Stirling engine by an optimal motion of its two independent pistons. Of particular interest is the dependence of the performance features on the extent of regeneration possible in the imperfect regenerator. Our results are based on an endoreversible model of the Stirling engine capturing the most important dissipation processes. More specifically, we determine for cyclic operation that volume dynamics out of the AS class of piston motions, for which the cycle averaged power output of the Stirling engine is maximized. In particular, for given system parameters $\alpha, \beta, \kappa$ and the regeneration coefficients $r_{1}, r_{2}$ and for the motion parameters $\sigma_{1}, \delta_{1}, \sigma_{2}, \delta_{2}$ and $\Delta t$ the dynamical equations are integrated until cyclic operation is reached. Then the power output and other quantities relevant for the analysis are determined.

By varying the motion parameters and repeating the procedure the power output is optimized numerically based on a Nelder-Mead approach [58]; the corresponding results are labeled OS (optimized sinusoidal). For comparison the standard piston motion labeled ST will be used.

In our investigation of the Stirling engine performance we will look at three different cases distinguished by their parameter sets. For the reference case the system parameters are chosen to be: $T_{\mathrm{H}}=400{ }^{\circ} \mathrm{C}, T_{\mathrm{C}}=300{ }^{\circ} \mathrm{C}, t_{0}=0.1 \mathrm{~s}, n_{0}=2 \mathrm{~mol}, V_{\text {dead }}=0.5 \mathrm{~L}, \Delta V=5 \mathrm{~L}, \beta_{0}=25 \mathrm{kJs} / \mathrm{m}^{6}$, $\alpha_{0}=500 \mathrm{~mol} /(\mathrm{s} \mathrm{bar}), \kappa_{0}=1.4 \times 10^{5} \mathrm{~W} / \mathrm{K}$. With these choices the reference case is characterized by a relatively large heat conductance and mass transfer. The other two cases are chosen for demonstrating the impact of a small heat conductance and small mass transfer coefficient. The low conductance case has the same parameters as the reference case but $\kappa_{<}=\kappa_{0} / 20$. The low mass transfer case has the same parameters as the reference case but $\alpha_{<}=\alpha_{0} / 20$. 
For these three cases the engine performance is studied in particular as a function of the regeneration coefficient $r=\left(r_{1}+r_{2}\right) / 2$ in the range $0 \leq r \leq 0.95$. This range ensures that $r_{1}$ and $r_{2}$ are both smaller than one and that the cycle averaged regeneration-related entropy production rate is positive.

\subsection{Power}

In Figures 4 and 5 the optimized power output is shown for the reference case in comparison with the low mass transfer and low conductance cases, respectively. For the reference case the OS motion shows a power output of about $150 \%$ of the ST motion. This is a quite remarkable increase. Even more remarkable is the fact that this power increase is nearly independent of the regeneration coefficient $r$ over its full range. The same applies to the performance of the low mass transfer case shown in Figure 4. Again it shows nearly no $r$ dependence, however the relative power gain is much larger than for the reference case.

In order to understand that behavior one has to consider the effects of the regeneration rate $r$. With little regeneration (corresponding to small $r$ ) most of the heat leaving the hot cylinder will reach the cold one and will be transported further into the cold bath. When the gas flow is reversed the cold gas will not be heated up much and would cool down the gas in the hot cylinder unless the connectivity to the hot bath is large enough to compensate that effect. So for a large heat conductance as in the reference case the effects of little regeneration are mitigated by increased heat fluxes from or to the heat baths. This can be seen in Figure 5, where the power output for the low conductance case declines with smaller $r$.

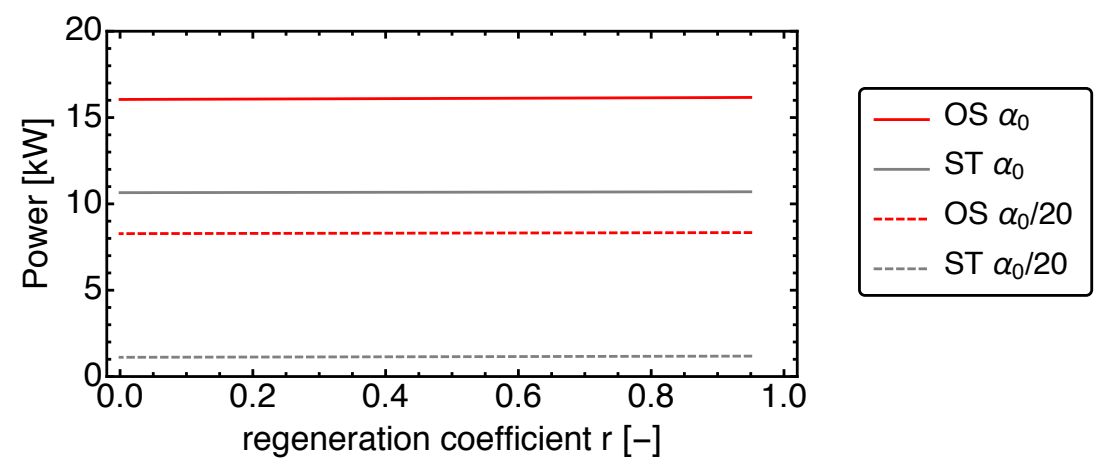

Figure 4. The power output is shown as a function of the regeneration coefficient $r$ for the reference case in comparison with the low mass transfer case. For both, the power output is nearly unaffected by the extent of the regeneration independent of the used motion.

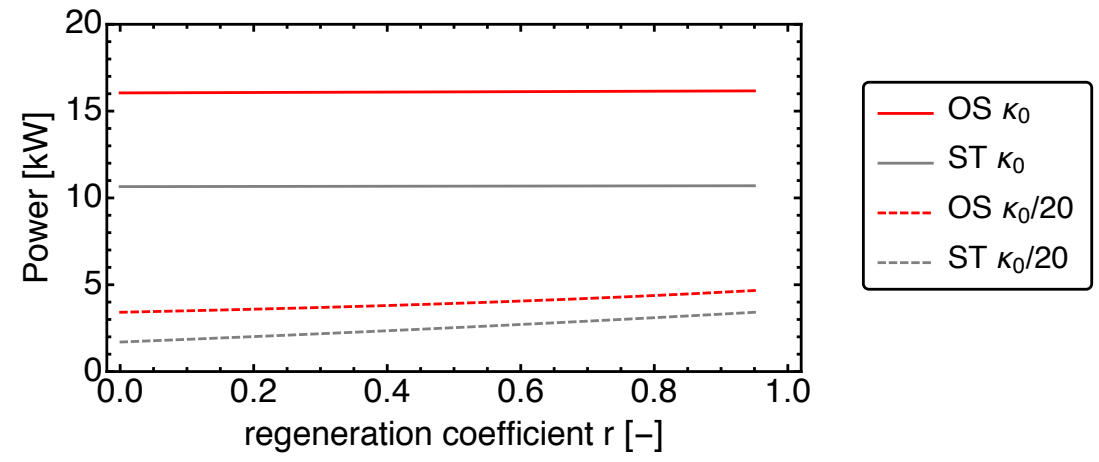

Figure 5. The power output is shown as a function of the regeneration coefficient $r$ for the reference case in comparison with the low conductance case. While the power output for the reference case is nearly unaffected by the extent of the regeneration this is different for the low conductance case, which shows a declining power with declining regeneration. 


\subsection{Efficiency}

While on the one hand the power output is little affected for a large enough heat conductance, the efficiency on the other hand is affected quite a bit: The increased heat fluxes needed for the maximum power operation get larger and larger the smaller the regeneration coefficient gets. Figures 6 and 7 show this clearly. As a reference value for the efficiency one can take the Carnot efficiency $\eta_{\text {Carnot }}=0.25$ for the given heat bath temperatures.

In the reference case the efficiency declines for the OS motion from about $21 \%$ at $95 \%$ regeneration down to roughly $13 \%$ for no regeneration, and for the ST motion from about $21 \%$ to below $12 \%$. The fact that both curves cross demonstrate that it is not the efficiency that is optimized but the power output. In the low mass transfer case the efficiency is considerably lower; from below $20 \%$ it declines down to $9 \%$ for the OS motion and is in the $1 \%$ to $3 \%$ range for the ST motion.

For the low conductance case the efficiency declines as well. However, the decline is steeper compared to the low mass transfer case, as a comparison of Figures 6 and 7 reveals: from a starting value at $95 \%$ regeneration at about half the Carnot efficiency it goes down to slightly below $5 \%$. Interestingly, the efficiency for the ST motion is larger than for the low mass transfer case starting at more than $7 \%$. However, once it reaches the no regeneration region it also shows the very low efficiency of the low mass transfer case.

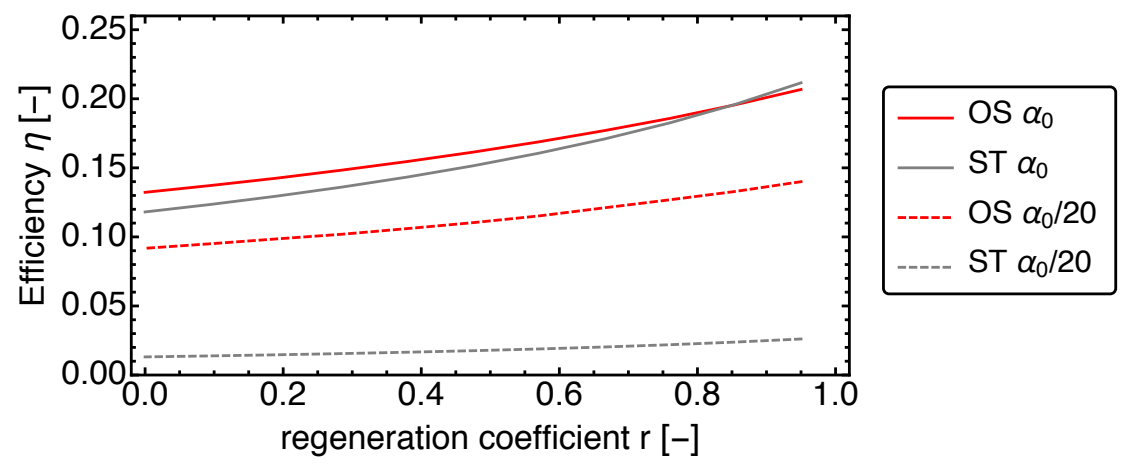

Figure 6. The efficiency is shown as a function of the regeneration coefficient $r$ for the reference case in comparison with the low mass transfer case. For both cases the efficiency is clearly decaying with weaker regeneration. Moreover the efficiency for the standard motion is larger than for the OS motion close to perfect regeneration for the reference case. Note that the efficiency for the OS motion is improved by factors on the order of 5 compared to the ST motion for the low mass transfer case.

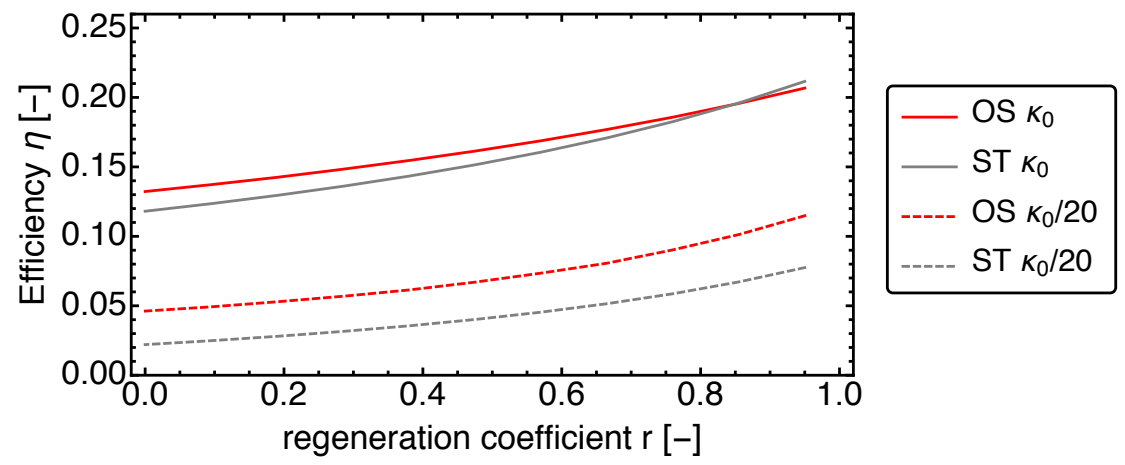

Figure 7. The efficiency is shown as a function of the regeneration coefficient $r$ for the reference case in comparison with the low conductance case. For both cases the efficiency is clearly decaying with weaker regeneration. Again the efficiency for the standard motion is larger than for the OS motion close to perfect regeneration for the reference case. 


\subsection{Entropy Production}

Another interesting quantity to characterize the performance features is the cycle averaged entropy production rate $\Sigma$. It is displayed as a function of the regeneration coefficient in Figures 8 and 9 . Several observations can be made. For all three cases the entropy production rate decreases with increasing regeneration. That is not unexpected, as with better regeneration the amount of heat which has to pass the finite heat conduction is reduced and thus the entropy production also decreases.

For the reference case $\Sigma$ decays almost linearly with $r$, and moreover the absolute difference between the entropy production rates between the OS and the ST motion stays more or less constant. For the low mass transfer case the difference in $\Sigma$ between the OS and the ST motion is inverted: the standard motion has a higher entropy production than the optimized one. The same can be observed for the low conductance case. This feature would seem natural, as a higher entropy production reduces the ability to extract work from a given amount of heat, and thus a higher power output would require a reduced entropy production. However the reference case shows, that this need not be true: the OS motion can achieve a higher output "paying an entropy production toll" for its performance.

Finally it is worth noting that for the low mass transfer case the entropy production rate is higher than for the low conductance case. This indicates that the finite mass transfer through the regenerator leads to a larger pressure difference, which in turn gives a higher difference in the chemical potential. That finally leads to an increased entropy production because the gas entering the receiving cylinder differs not only in temperature but also in a significant fashion with respect to pressure from the receiving gas.

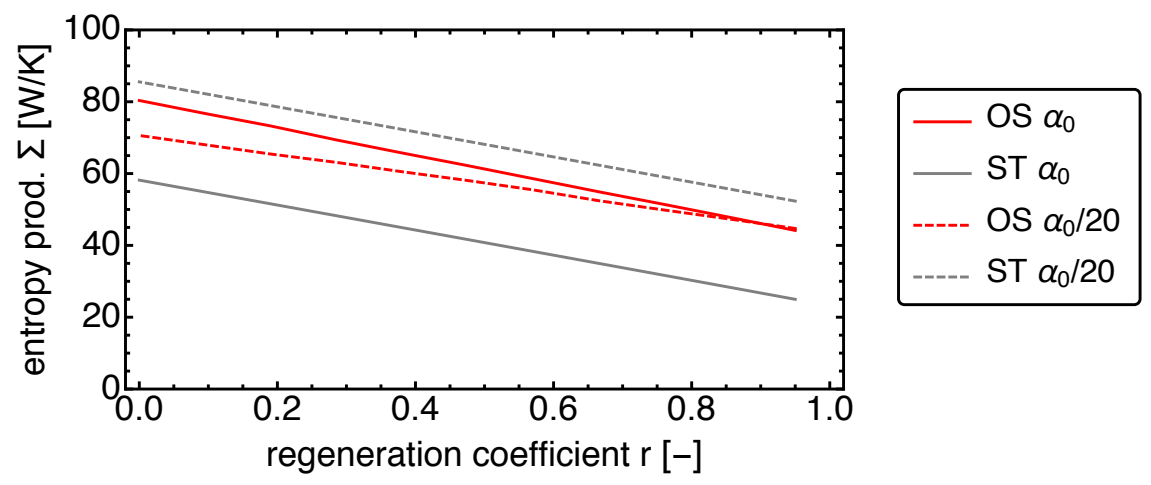

Figure 8. The cycle averaged entropy production rate is shown as a function of the regeneration coefficient $r$ for the reference case in comparison with the low mass transfer case. For both cases the entropy production increases considerably with weaker regeneration.

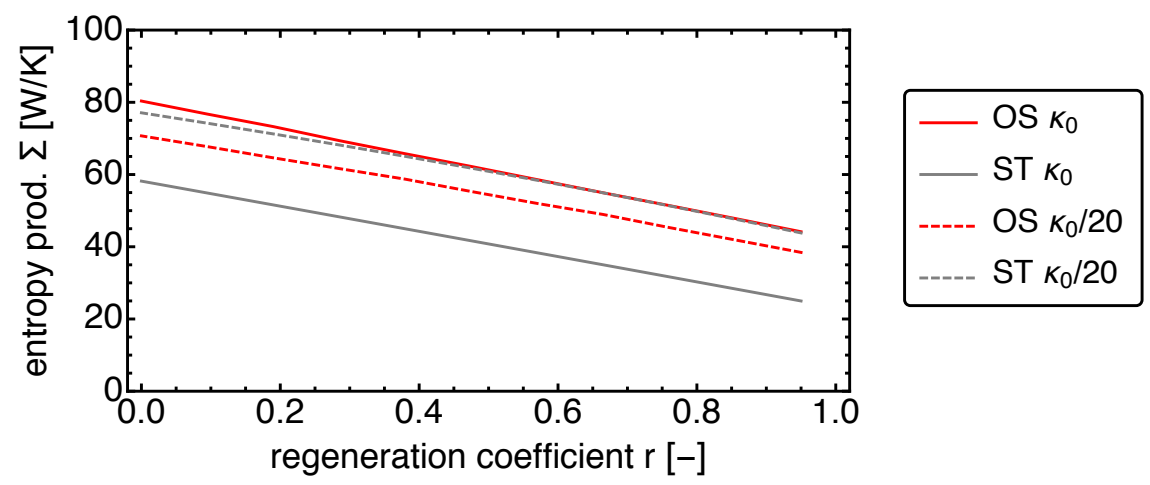

Figure 9. The cycle averaged entropy production rate is shown as a function of the regeneration coefficient $r$ for the reference case in comparison with the low conductance case. For the reference case the OS motion leads to a stronger increase in the entropy production than for the low conductance case. 


\subsection{OS Piston Motion}

Finally we show the OS piston motion for the three cases introduced above for two different regeneration situations: a very good regeneration at $r=0.9$ and a very weak regeneration at $r=0.1$. In Figure 10 the temporal behavior of $V_{1}(t)$ is shown, in Figure 11 that of $V_{2}(t)$. The first observation in both figures is that the OS motion is clearly different from the ST motion for all three cases and for both regeneration situations. A surprising result is that for all three cases the $10 \%$ regeneration and the $90 \%$ regeneration lead to very similar piston motions.

For both volumes the largest deviations can be observed for the low conductance case. All trajectories show a trapezoid wave form, where the reference case has the most noticeable one, while the other cases are closer to a sine wave form. Also the time shift between the two volumes clearly depends on the case. Overall the degrees of freedom provided by the AS piston motion class are well used by the different cases, while the influence of the regeneration extent is of minor importance.
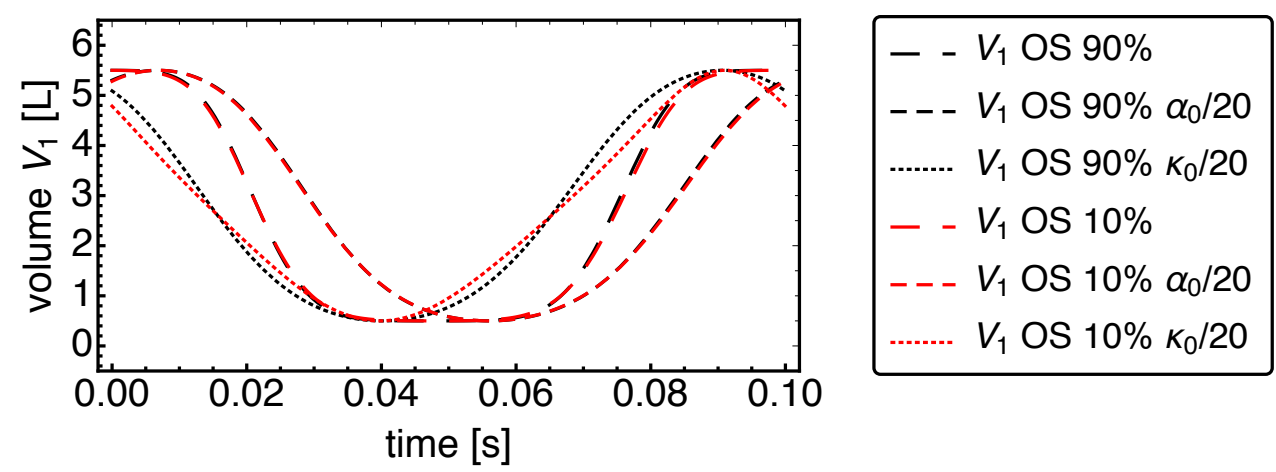

Figure 10. The optimized volume dynamics of the hot cylinder is shown as a function of time for the reference, low mass transfer and the low conductance cases with $r=10 \%$ and $r=90 \%$. The influence of the regeneration coefficient is clearly visible, even though the impact of the different cases is much larger.
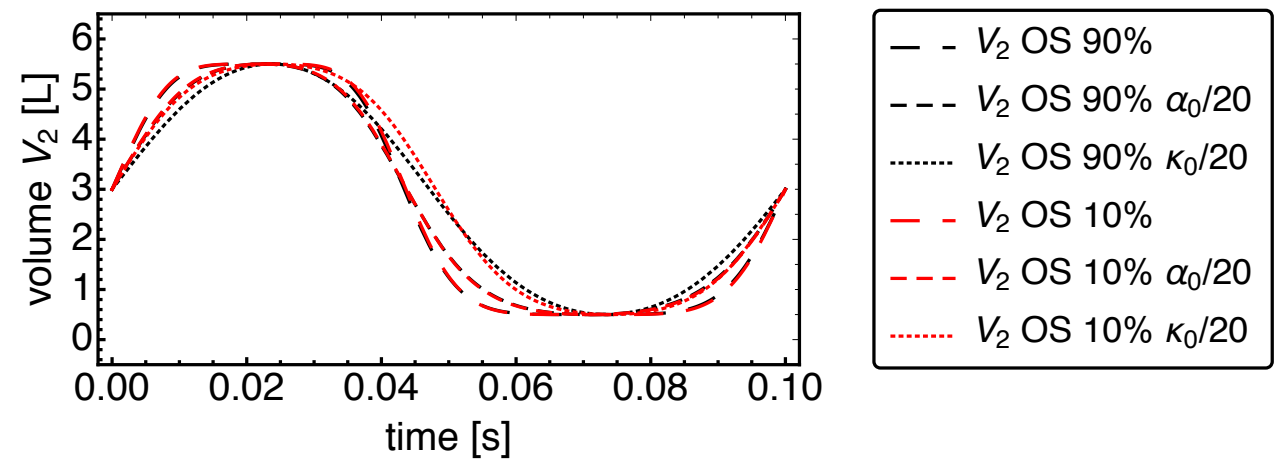

Figure 11. The optimized volume dynamics of the cold cylinder is shown as a function of time for the reference, low mass transfer and the low conductance cases with $r=10 \%$ and $r=90 \%$. The influence of the regeneration coefficient is clearly visible, but the impact of the different cases is considerably smaller than for the hot cylinder.

\section{Conclusions}

Within this work, possible performance improvements were investigated for an alpha-type Stirling engine by optimizing its piston motion. To limit optimization complexity, the possible motion paths of each of both pistons were chosen from a particular functional class introduced as adjustable sinusoidal (AS) motion. This motion is governed by two parameters for each piston and one phase shift parameter. Thus, the complete optimization space consists of only five parameters. Additionally, the particular choice of the AS motion class is motivated by previous optimization results [27], which show that 
letting the piston rest at extreme positions for a while on the one hand and unevenly distributing these resting times between both extreme positions on the other hand has proven to be beneficial for the thermal process efficiency.

The primary aim of this analysis is to understand the impact of imperfect regeneration within the Stirling engine upon the engines power output and efficiency. To this end, a mathematical model based on Endoreversible Thermodynamics has been developed, which particularly includes the main dissipative process of a non-ideal regenerator represented by a single parameter, the regeneration coefficient $r$.

In particular, we have set up three distinct cases where we analyzed the engines power output, efficiency and entropy generation for both standard and optimized motion paths while varying the regeneration coefficient. These cases are a reference case, a case with low mass transfer and one with low heat conduction. In all these cases our results confirm the role of the regenerator as an efficiency enhancing component for the Stirling engine for both standard and optimized motion paths. The power output in the base case is largely independent from the regeneration coefficient while for reduced mass transfer or heat conductance, the power output increases with better regeneration. The efficiency is enhanced by a larger regeneration coefficient in any case.

Additionally, it was also observed that optimizing for maximal power output may lead to a decreased efficiency, as maximal power output and maximal efficiency are distinct operation points [47].

Summarizing, the maximal power output of the considered Stirling engine model benefits largely from the motion path optimization. Especially in cases with limited mass transfer or heat conductance, it benefits from an improved regeneration as well. The optimization for maximal power output may however cause a decrease in efficiency. In certain application scenarios like waste heat recovery, where the thermal energy supply is effectively for free, this can be desirable as the engine needs to pay itself off by the power generated.

The above findings can provide the basis for future research efforts. An important next step on the theoretical side of investigations would be a full control theoretic analysis of the optimized piston movement. It would be very interesting to see to what extent the AS motion has exhausted the optimization potential for the cycle averaged power output. On the practical side it would of course be important to investigate to what extent the optimized piston motion can be approximated by appropriate piston drives. For that purpose one can use mechanical couplings, but also electrical motor driven pistons are possible, for which an appropriate excitation control in combination with the inertial forces can—within limits—generate the desired motion.

Author Contributions: All authors contributed equally to this article. All authors have read and agreed to the published version of the manuscript

Funding: This research has been funded by the German Federal Ministry of Education and Research under support code 01LY1218C. The publication of this article was funded by Chemnitz University of Technology.

Conflicts of Interest: The authors declare no conflict of interest.

\section{Nomenclature}

Greek symbols:

$\begin{array}{ll}\Delta \phi & \text { Piston phase shift } \\ \Delta t & \text { Piston time shift } \\ \alpha & \text { Mass transfer coefficient } \\ \beta & \text { Mechanical friction coefficient } \\ \delta, \sigma & \text { Motion control parameter } \\ \eta & \text { Efficiency } \\ \mathcal{K} & \text { Heat conductance } \\ \mu & \text { Chemical potential }\end{array}$




\begin{tabular}{|c|c|}
\hline \multicolumn{2}{|l|}{ Symbols: } \\
\hline$E$ & Energy \\
\hline$I$ & Flux of energy \\
\hline$J$ & Flux of extensity \\
\hline$P, P_{\text {out }}$ & Power \\
\hline$R$ & Gas constant \\
\hline$S$ & Entropy \\
\hline$T$ & Temperature \\
\hline$U$ & Internal energy \\
\hline$V$ & Cylinder volume \\
\hline$V_{\text {dead }}$ & Cylinder dead volume \\
\hline$X$ & Extensity \\
\hline Y & Intensity \\
\hline$\hat{c}_{V}$ & Specific heat capacity \\
\hline$h$ & Molar enthalpy \\
\hline$n$ & Mole number \\
\hline$p$ & Pressure \\
\hline$r$ & Regeneration coefficient \\
\hline$t$ & Time \\
\hline$t_{0}$ & Period of motion \\
\hline$q$ & Heat flux \\
\hline \multicolumn{2}{|l|}{ Subscripts: } \\
\hline 0 & Reference \\
\hline 1,2 & Piston number \\
\hline$C, c$ & Cold \\
\hline $\mathrm{H}, \mathrm{h}$ & Hot \\
\hline e & Environment \\
\hline $\mathrm{f}$ & Friction \\
\hline$i$ & Subsystem \\
\hline$k$ & Contact point \\
\hline $\mathrm{r}$ & Regenerator \\
\hline \multicolumn{2}{|l|}{ Superscripts: } \\
\hline$\alpha$ & Extensity type \\
\hline \multicolumn{2}{|l|}{ Abbreviations: } \\
\hline AS & Adjustable sinusoidal motion \\
\hline OS & Optimized sinusoidal motion \\
\hline ST & Standard harmonic motion \\
\hline SR & Entropy reservoir \\
\hline WR, WT, WF & Work reservoirs \\
\hline
\end{tabular}

\section{References}

1. Stirling, R. Stirling Air Engine and the Heat Regenerator. British Patent 4081, 16 November 1816.

2. Reader, G.T. Stirling Regenerators. Heat Transf. Eng. 1994, 15, 19-25. [CrossRef]

3. Timoumi, Y.; Tlili, I.; Ben Nasrallah, S. Design and performance optimization of GPU-3 Stirling engines. Energy 2008, 33, 1100-1114. [CrossRef]

4. Duan, C.; Wang, X.; Shu, S.; Jing, C.; Chang, H. Thermodynamic design of Stirling engine using multi-objective particle swarm optimization algorithm. Energ. Convers. Manag. 2014, 84, 88-96. [CrossRef]

5. Hooshang, M.; Askari Moghadam, R.; Alizadeh Nia, S.; Tale Masouleh, M. Optimization of Stirling engine design parameters using neural networks. Renew. Energy 2015, 74, 855-866. [CrossRef]

6. Ferreira, A.C.; Teixeira, S.; Teixeira, J.C.; Martins, L.B. Design Optimization of a Solar Dish Collector for Its Application With Stirling Engines. In Proceedings of the ASME 2015 International Mechanical Engineering Congress and Exposition, Houston, TX, USA, 13-19 November 2015. [CrossRef]

7. Sowale, A.; Kolios, A.J. Thermodynamic Performance of Heat Exchangers in a Free Piston Stirling Engine. Energies 2018, 11, 505. [CrossRef] 
8. Sowale, A.; Anthony, E.J.; Kolios, A.J. Optimisation of a Quasi-Steady Model of a Free-Piston Stirling Engine. Energies 2019, 12, 72. [CrossRef]

9. Mozurkewich, M.; Berry, R.S. Finite-time thermodynamics: Engine performance improved by optimized piston motion. Proc. Natl. Acad. Sci. USA 1981, 78, 1986-1988. [CrossRef]

10. Hoffmann, K.H.; Watowich, S.J.; Berry, R.S. Optimal Paths for Thermodynamic Systems: The Ideal Diesel Cycle. J. Appl. Phys. 1985, 58, 2125-2134. [CrossRef]

11. Huleihil, M.; Andresen, B. Optimal piston trajectories for adiabatic processes in the presence of friction. J. Appl. Phys. 2006, 100, 114914. [CrossRef]

12. Huleihil, M. Optimal Stroke Path for Reciprocating Heat Engines. Modell. Simul. Eng. 2019, $2019,7468478$. [CrossRef]

13. Chen, L.; Ma, K.; Ge, Y.; Feng, H. Re-Optimization of Expansion Work of a Heated Working Fluid with Generalized Radiative Heat Transfer Law. Entropy 2020, 22, 720. [CrossRef]

14. Mozurkewich, M.; Berry, R.S. Optimal Paths for Thermodynamic Systems: The ideal Otto Cycle. J. Appl. Phys. 1982, 53, 34-42. [CrossRef]

15. Xia, S.; Chen, L.; Sun, F. Maximum power configuration for multireservoir chemical engines. J. Appl. Phys. 2009, 105, 1-6. [CrossRef]

16. Ge, Y.; Chen, L.; Sun, F. Optimal path of piston motion of irreversible Otto cycle for minimum entropy generation with radiative heat transfer law. J. Energy Inst. 2012, 85, 140-149. [CrossRef]

17. Chen, L.; Xia, S.; Sun, F. Optimizing piston velocity profile for maximum work output from a generalized radiative law Diesel engine. Math. Comput. Model. 2011, 54, 2051-2063. [CrossRef]

18. Xia, S.; Chen, L.; Sun, F. Engine performance improved by controlling piston motion: Linear phenomenological law system Diesel cycle. Int. J. Therm. Sci. 2012, 51, 163-174. [CrossRef]

19. Lin, J.; Chang, S.; Xu, Z. Optimal motion trajectory for the four-stroke free-piston engine with irreversible Miller cycle via a Gauss pseudospectral method. J. Non-Equilib. Thermodyn. 2014, 39, 159-172. [CrossRef]

20. Kojima, S. Theoretical Evaluation of the Maximum Work of Free-Piston Engine Generators. J. Non-Equilib. Thermodyn. 2017, 42, 31-58. [CrossRef]

21. Watowich, S.J.; Hoffmann, K.H.; Berry, R.S. Optimal Paths for a Bimolecular, Light-Driven Engine. Il Nuovo Cim. B 1989, 104, 131-147. [CrossRef]

22. Ma, K.; Chen, L.; Sun, F. Optimal paths for a light-driven engine with a linear phenomenological heat transfer law. Sci. China. Chem. 2010, 53, 917-926. [CrossRef]

23. Chen, L.; Ma, K.; Ge, Y. Optimal Configuration of a Bimolecular, Light-Driven Engine for Maximum Ecological Performance. Arab. J. Sci. Eng. 2017, 38, 341-350. [CrossRef]

24. Kojima, S. Maximum Work of Free-Piston Stirling Engine Generators. J. Non-Equilib. Thermodyn. 2017, 42, 169-186. [CrossRef]

25. Craun, M.; Bamieh, B. Optimal Periodic Control of an Ideal Stirling Engine Model. J. Dyn. Syst. Meas. Control 2015, 137, 071002. [CrossRef]

26. Craun, M.J. Modeling and Control of an Actuated Stirling Engine. Ph.D Thesis, University of California, Santa Barbara, CA, USA, 2015. Available online: https:// escholarship.org/uc/item/2tk2v9kj (accessed on 1 September 2020).

27. Masser, R.; Khodja, A.; Scheunert, M.; Schwalbe, K.; Fischer, A.; Paul, R.; Hoffmann, K.H. Optimized Piston Motion for an Alpha-Type Stirling Engine. Entropy 2020, 22, 700. [CrossRef]

28. Andresen, B.; Berry, R.S.; Nitzan, A.; Salamon, P. Thermodynamics in Finite Time. I. The Step-Carnot Cycle. Phys. Rev. A 1977, 15, 2086-2093. [CrossRef]

29. Salamon, P.; Andresen, B.; Berry, R.S. Thermodynamics in Finite Time. II. Potentials for Finite-Time Processes. Phys. Rev. A 1977, 15, 2094-2102. [CrossRef]

30. Andresen, B.; Salamon, P.; Berry, R.S. Thermodynamics in finite time: Extremals for imperfect heat engines. J. Chem. Phys. 1977, 66, 1571-1577. [CrossRef]

31. Andresen, B.; Salamon, P.; Berry, R.S. Thermodynamics in Finite Time. Phys. Today 1984, 37, 62-70. [CrossRef]

32. Hoffmann, K.H.; Burzler, J.M.; Schubert, S. Endoreversible Thermodynamics. J. Non-Equilib. Thermodyn. 1997, 22, 311-355.

33. Hoffmann, K.H.; Burzler, J.M.; Fischer, A.; Schaller, M.; Schubert, S. Optimal Process Paths for Endoreversible Systems. J. Non-Equilib. Thermodyn. 2003, 28, 233-268. [CrossRef] 
34. Hoffmann, K.H. An introduction to endoreversible thermodynamics. AAPP Phys. Math. Nat. Sci. 2008, 86, 1-19. [CrossRef]

35. Rubin, M.H. Optimal Configuration of a Class of Irreversible Heat Engines. I. Phys. Rev. A 1979, 19, 1272-1276. [CrossRef]

36. Rubin, M.H.; Andresen, B. Optimal Staging of Endoreversible Heat Engines. J. Appl. Phys. 1982, 53, 1-7. [CrossRef]

37. De Vos, A. Reflections on the power delivered by endoreversible engines. J. Phys. D Appl. Phys. 1987, 20, 232-236. [CrossRef]

38. Chen, J.; Yan, Z. Optimal Performance of an Endoreversible-Combined Refrigeration Cycle. J. Appl. Phys. 1988, 63, 4795-4798. [CrossRef]

39. Bădescu, V. On the Theoretical Maximum Efficiency of Solar-Radiation Utilization. Energy 1989, 14, 571-573. [CrossRef]

40. De Vos, A. Is a solar cell an edoreversible engine? Sol. Cells 1991, 31, 181-196. [CrossRef]

41. Schwalbe, K.; Hoffmann, K.H. Optimal Control of an Endoreversible Solar Power Plant. J. Non-Equilib. Thermodyn. 2018, 43, 255-271. [CrossRef]

42. Schwalbe, K.; Hoffmann, K.H. Novikov engine with fluctuating heat bath temperature. J. Non-Equilib. Thermodyn. 2018, 43, 141-150. [CrossRef]

43. Andresen, B.; Salamon, P. Distillation by Thermodynamic Geometry. In Thermodynamics of Energy Conversion an Transport; Sieniutycz, S.; De Vos, A., Eds.; Springer: New York, NY, USA, 2000; Chapter 12, pp. 319-331.

44. Wagner, K.; Hoffmann, K.H. Endoreversible modeling of a PEM fuel cell. J. Non-Equilib. Thermodyn. 2015, 40, 283-294. [CrossRef]

45. Tsirlin, A.; Sukin, I.A.; Balunov, A.; Schwalbe, K. The Rule of Temperature Coefficients for Selection of Optimal Separation Sequence for Multicomponent Mixtures in Thermal Systems. J. Non-Equilib. Thermodyn. 2017, 42, 359-369. [CrossRef]

46. Marsik, F.; Weigand, B.; Thomas, M.; Tucek, O.; Novotny, P. On the Efficiency of Electrochemical Devices from the Perspective of Endoreversible Thermodynamics. J. Non-Equilib. Thermodyn. 2019, 44, 425-437. [CrossRef]

47. Fischer, A.; Hoffmann, K.H. Can a quantitative simulation of an Otto engine be accurately rendered by a simple Novikov model with heat leak? J. Non-Equilib. Thermodyn. 2004, 29, 9-28. [CrossRef]

48. Ding, Z.; Chen, L.; Sun, F. Finite time exergoeconomic performance for six endoreversible heat engine cycles: Unified description. Appl. Math. Mod. 2011, 35, 728-736. [CrossRef]

49. Paéz-Hernández, R.T.; Chimal-Eguía, J.C.; Sánchez-Salas, N.; Ladino-Luna, D. General Properties for an Agrowal Thermal Engine. J. Non-Equilib. Thermodyn. 2018, 43, 131-139. [CrossRef]

50. Masser, R.; Hoffmann, K.H. Dissipative Endoreversible Engine with Given Efficiency. Entropy 2019, $21,1117$. [CrossRef]

51. Açıkkalp, E.; Yamık, H. Modeling and optimization of maximum available work for irreversible gas power cycles with temperature dependent specific heat. J. Non-Equilib. Thermodyn. 2015, 40, 25-39. [CrossRef]

52. Masser, R.; Hoffmann, K.H. Endoreversible Modeling of a Hydraulic Recuperation System. Entropy 2020, 22, 383. [CrossRef]

53. De Vos, A. Endoreversible Models for the Thermodynamics of Computing. Entropy 2020, 22, 660. [CrossRef]

54. Schwalbe, K.; Hoffmann, K.H. Stochastic Novikov Engine with Fourier Heat Transport. J. Non-Equilib. Thermodyn. 2019, 44, 417-424. [CrossRef]

55. Curzon, F.L.; Ahlborn, B. Efficiency of a Carnot Engine at Maximum Power Output. Am. J. Phys. 1975, 43, 22-24. [CrossRef]

56. Wagner, K.; Hoffmann, K.H. Chemical reactions in endoreversible thermodynamics. Eur. J. Phys. 2016, 37, 015101. [CrossRef]

57. Kuehl, H.D.; Schulz, S. A 2nd order regenerator model including flow dispersion and bypass losses. In Proceedings of the IECEC 96, 31st Intersociety Energy Conversion Engineering Conference, Washington, DC, USA, 11-16 August 1996; Volume 2, pp. 1343-1348.

58. Nelder, J.A.; Mead, R. A Simplex Method for Function Minimization. Comput. J. 1965, 7, 308-313. [CrossRef]

(C) 2020 by the authors. Licensee MDPI, Basel, Switzerland. This article is an open access article distributed under the terms and conditions of the Creative Commons Attribution (CC BY) license (http:/ / creativecommons.org/licenses/by/4.0/). 